\title{
Formalism of Biological Tissues/Nanowire Sensor Interface Behavior
}

\author{
MARWA SAWAN \\ Institute of Electronics, Microelectronics and Nanotechnology, Opto-Acousto-Electronic Department \\ UniversityPolytechnic Hauts-De-France \\ Campus Mont Houy - 59313 Valenciennes Cedex 9 \\ FRANCE
}

\author{
HILAL REDA \\ Faculty of Engineering \\ Lebanese University \\ Hadath Campus \\ LEBANON \\ NADINE SAAD \\ Faculty of Science \\ Lebanese University \\ Fanar Campus \\ LEBANON \\ SUN BIN \\ College of Physics \\ Qingdao University \\ Qingdao 266071 \\ P.R CHINA
}
GEORGES NASSAR
Institute of Electronics, Microelectronics and Nanotechnology, Opto-Acousto-Electronic Department UniversityPolytechnic Hauts-De-France
Campus Mont Houy - 59313 Valenciennes Cedex 9
FRANCE

\section{Marwa.Sawan@etu.uphf.fr}

\begin{abstract}
In this paper, we investigate the behaviour of biological tissues (skin) coupled to a flexible sensor embedded at a solid substrate based on a numerical model taking into account the relationship between strain/stress components at the interface. Based on this study, the most appropriate biomechanical factors are understood and quantified in order to optimize the sensor/biological tissue interface conditions. A micromechanical description based on a mathematical formulation has been developed to evaluate the biomechanical behaviour provided by a 2D viscoelastic model of Kelvin-Voigt. Based on the results, it appears that the model can be used effectively to characterize in-vivo the dynamic properties of soft tissues in order to adapt the biophysical properties of flexible sensors dedicated to optimal adhesion.
\end{abstract}

Key-Words: -Biological tissues- sensor - substrate - interface- biomechanical behavior- Kelvin-Voigt- adhesion.

Received: September 23, 2019. Revised: January 3, 2020. Accepted: February 5, 2020. Published: February 24, 2020.

\section{Introduction}

The evolution of the population poses new challenges into our system of "Self-Dynamics" to meet the needs of citizens in a similar way around the globe. The study of interactions between sensors and living fields goes far beyond biological and health impact studies. The many medical challenges, in the context of the development or application of treatments, are at the origin of potential innovations in the areas that we intend to explore.

In the field of tissue engineering, cell compatibility for a scaffold has a major influence on the cell 
growth and the development of new synthetic tissues with a very low risk of infection [1-3]. However, cell-biomaterial interactions are a very complex phenomenon and in order to optimize these interactions, it is important to understand how cells interact with artificial surfaces. It has been well demonstrated that numerous parameters influence the interfaces characteristics; these include the nature of the biomaterial [4] and its surface characteristics, such as roughness [5-7]. In the multilayer tissues human case, a question that arises at, in dynamic mode, how the involved layers interact with each other? Hence, an established method for modelling the dynamic motion in a multilayer have been developed from formulations that combine the physical and mechanical characteristics of each layer with the interaction conditions of the waves at the interfaces between every two adjacent layers, or the so-called boundary conditions such as skin/sensor.

In order to create sensitive interfaces between the skin and the substrate (exoskeleton), a part of this study will be dedicated to the characterization of flexible and extensible nano-wire sensors whose design is based on ultra-fine electrospun fibers.

\section{Tissue Interface}

\subsection{Numerical Approach}

In order to quantify the response of the skin, a micromechanical model based on a finite element homogenization method is used under both static and harmonic applied loads.

Indeed, the creep function associated to a pure normal stress is computed by finite element simulation of a two-dimensional Kelvin-Voigt (K$\mathrm{V})$ viscoelastic model. The general constitutive law for $2 \mathrm{D} \mathrm{K}-\mathrm{V}$ is given by:

$$
\begin{aligned}
{\left[\begin{array}{c}
\sigma_{\mathrm{xx}} \\
\sigma_{\mathrm{yy}} \\
\sigma_{\mathrm{xy}}
\end{array}\right]=\left(\begin{array}{ccc}
\lambda+2 \mu & \mu & 0 \\
\mu & \lambda+2 \mu & 0 \\
0 & 0 & 2 \mu
\end{array}\right)\left(\begin{array}{c}
\varepsilon_{\mathrm{xx}} \\
\varepsilon_{\mathrm{yy}} \\
\varepsilon_{\mathrm{xy}}
\end{array}\right) } \\
+\left(\begin{array}{ccc}
\eta_{\mathrm{v}}+2 \eta_{\mathrm{s}} & \eta_{\mathrm{s}} & 0 \\
\eta_{\mathrm{s}} & \eta_{\mathrm{v}}+2 \eta_{\mathrm{s}} & 0 \\
0 & 0 & 2 \eta_{\mathrm{s}}
\end{array}\right)\left(\begin{array}{c}
\dot{\varepsilon}_{\mathrm{xx}} \\
\dot{\varepsilon}_{\mathrm{yy}} \\
\dot{\varepsilon}_{\mathrm{xy}}
\end{array}\right)
\end{aligned}
$$

Where $\lambda, \mu$ are the Lamé coefficients, and $\eta_{v}, \eta_{s}$ are the volume and shear viscosity, respectively. When applying constant vertical shear $\operatorname{stress} \sigma=$

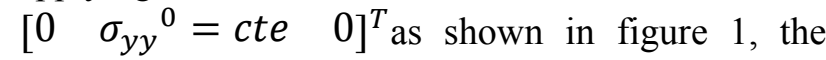
creep functions under vertical constant load will be as written in equation (2).

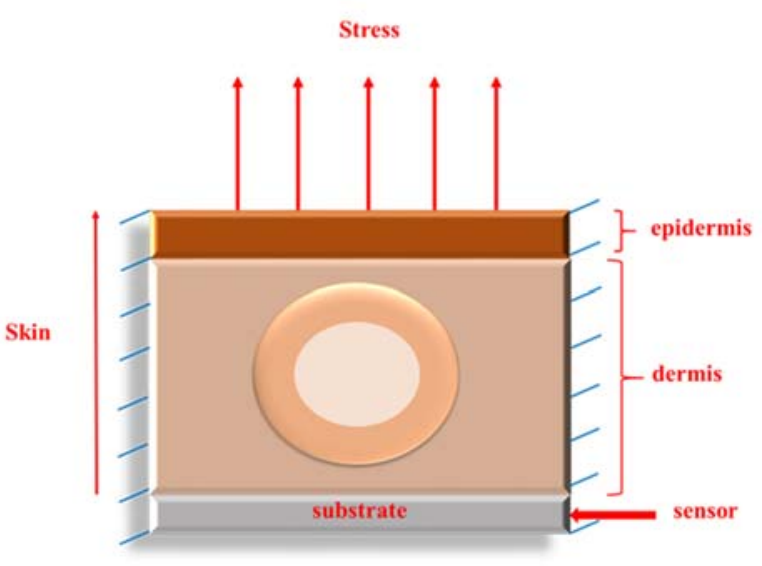

(a)

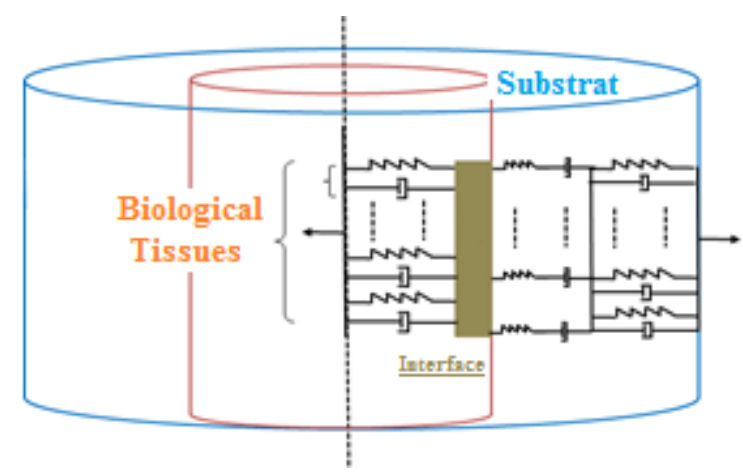

(b)

Fig.1 Applied vertical stress on the corresponding unit cell; a) Finite element model; b) Mechanical generalized Kelvin-Voigt model.

$$
\begin{gathered}
\varepsilon_{x x}=-\frac{\sigma_{y y}}{4(\lambda+\mu)} e^{-\frac{(\lambda+\mu)}{\eta_{v}+\eta_{s}} t}+\frac{\sigma_{y y}}{4(\mu)} e^{-\frac{(\mu)}{\eta_{s}} t}-\frac{\sigma_{y y}}{4 \mu(\lambda+\mu)} ; \\
\varepsilon_{y y}=-\frac{\sigma_{y y}}{4(\lambda+\mu)} e^{-\frac{(\lambda+\mu)}{\eta_{v}+\eta_{s}} t}-\frac{\sigma_{y y}}{4(\mu)} e^{-\frac{(\mu)}{\eta_{s}} t}+\frac{(\lambda+2 \mu) \sigma_{y y}}{4 \mu(\lambda+\mu)} ; \\
\varepsilon_{x y}=0
\end{gathered}
$$

At $\mathrm{t}=\infty$, the deformation tends to horizontal asymptote of value $\frac{(\lambda+2 \mu) \sigma_{y y}}{4 \mu(\lambda+\mu)}$ at time $\mathrm{t}=5 \tau$. We present the calculation of the first Lamé coefficient $\lambda$ from the value of the maximum deformation according to equation 2 . Then, from the relation between $\lambda$ and $\mu$ given by $\frac{2 \mu \nu}{(1-2 v)}$, we can calculate the effective Poisson's ratio. The velocity of deformation is a function dependent on time. During the execution stage, the user must define an initial constraint, a step increment of time, as well as the characteristic of the material used (Young's modulus, Poisson's ratio, viscosity). Boundary conditions reflect the definition of the problem. In the calculation of nodal solutions, the software solves for the unknown displacement values with the given boundary conditions. The deformation can 
be calculated from the displacement history and the time step size. From the results of the Free FEM, the deformation was plotted as a function of time to characterize the creep response at short time.

In dynamic mode, we take a general 2D viscoelastic medium of $\mathrm{KV}$ under a normal harmonic stress $=\sigma_{0} e^{\omega t}$, where $\omega$ is the frequency. The general constitutive law for the $2 \mathrm{D} \mathrm{KV}$ is given by a coupling of the rigidity and viscosity matrices:

$$
\sigma(y, t)=\boldsymbol{a}(y) \varepsilon(y, t)+\boldsymbol{b}(y) \frac{\partial}{\partial t} \varepsilon(y, t)
$$

Where $\boldsymbol{a}(y)$ is the rigidity matrix, $\boldsymbol{b}(y)$ is the viscosity matrix. They represent the harmonic elastic behavior of the effective viscoelastic medium. Applying constant stress $\sigma_{0}$ and changing the frequency, we obtain the deformation as a function of the frequency.

\subsection{Strain Profile}

To validate the concept, this part of study uses known physical properties of the sensor (composite) and a skin cell formed of an epidermis and a dermis. In the model, the cell strain has an area of $12 \mathrm{~m} 2$ and two spheres of radius $4 \mathrm{~mm}$ and $3 \mathrm{~mm}$, respectively. The skin consists of a single incompressible homogeneous layer with linear viscoelastic, isotropic behavior. The study is carried out using $\mathrm{KV}$ model. A vertical normal stress is applied to the cell strain as shown in Fig. 1.

Table 1 shows the mechanical properties of substrate, epidermis, dermis composed of collagens, collamod, and membrane.

Table 1. The mechanical properties of substrate

\begin{tabular}{lccc}
\hline & $\mathbf{E}$ (MPa) & $\boldsymbol{\eta}(\mathbf{M P a . s})$ & $\boldsymbol{9}$ \\
\hline Epidermis & 0.06 & 0 & 0.3 \\
Collagens & 0.05 & 5000 & 0.4 \\
Collamod & 0.05 & 5000 & 0.39 \\
Membrane & 0.005 & 2 & 0.4 \\
composite & 94.103 & 0.4 & 0 \\
\hline
\end{tabular}

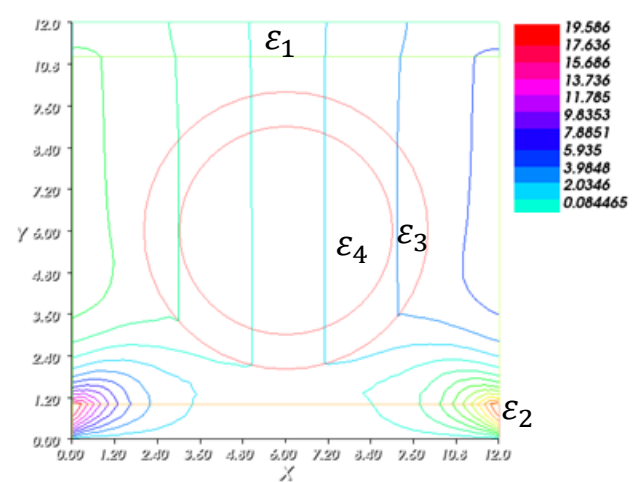

(a)

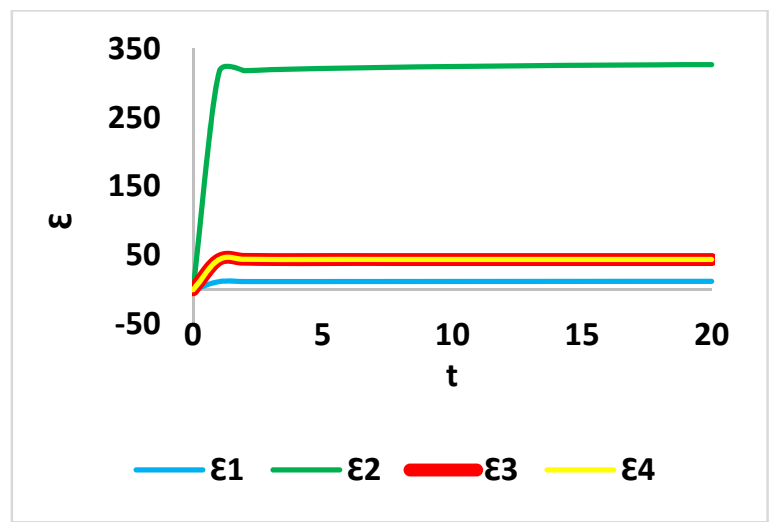

(b)

Fig.2Variation of the (a) displacement along the y-axis as a function of time showing the deformation at regions and (b) the deformation as a function of time for the four regions at $\mathrm{t}=20$ seconds and $\sigma=0.6 \mathrm{MPa}$.

The numerical approach (Free FEM) shows the internal deformations in different regions: $\varepsilon_{1}$ between the interface of the dermis and the epidermis, $\varepsilon_{2}$ between the interface sensor-skin cell, $\varepsilon_{3}$ nodule surface, and $\varepsilon_{4}$ the hole. As for the boundary conditions, a vertical stress is applied to the entire cell from region 3 with the interface of region 1 along the $\mathrm{x}$ and $\mathrm{y}$ axes. Figure 2 shows the deformation at different regions for $\mathrm{t}=20$ seconds and a normal stress of $\sigma=0.6 \mathrm{MPa}$.

These results show that there is a maximum deformation on the cell strain-substrate interface considered as a sensor. Even with a maximum stress, the maximum deformation remains in the elastic region. Thus, we can tear the skin without having any distortion inside. The variation of the deformation in function of time for the four regions is drawn in figure 2. 


\subsection{Embedded sensor}

\subsubsection{Thin layer interface sensor}

Tissue engineering is an interdisciplinary field involving different organs of man. One of the objectives remains the development of implantable systems or even in vivo reshaping of tissues. in the following, we will discuss the integration of nanowire sensors in tissues artificially designed forming part of a therapeutic/assistance physical support in order to optimize in vivo the comfort of people with a physical handicap of mobility or sensation. This "interface sensor" should have appropriate mechanical properties to provide a stress environment suitable for tissue behavior while respecting biocompatibility and current standards.

\subsubsection{Electro-spun nanowire concept}

Generally, lead zirconatetitanate (PZT) is a widelyused piezoelectric material with a high dielectric constant and piezoelectric voltage. However, the lead component is harmful to human health and makes this technology not compatible with public health applications. Therefore, as a substitute for PZT electromechanical component, a thin layer nano-wire composite (Figure 3) based on PVDF and barium titanate $\left(\mathrm{BaTiO}_{3}\right)$ is increasingly used for vibrational characteristics because of its excellent electromechanical properties [8]. This composite possesses a higher piezoelectric strain coefficient and thus can enable greater energy transfer.

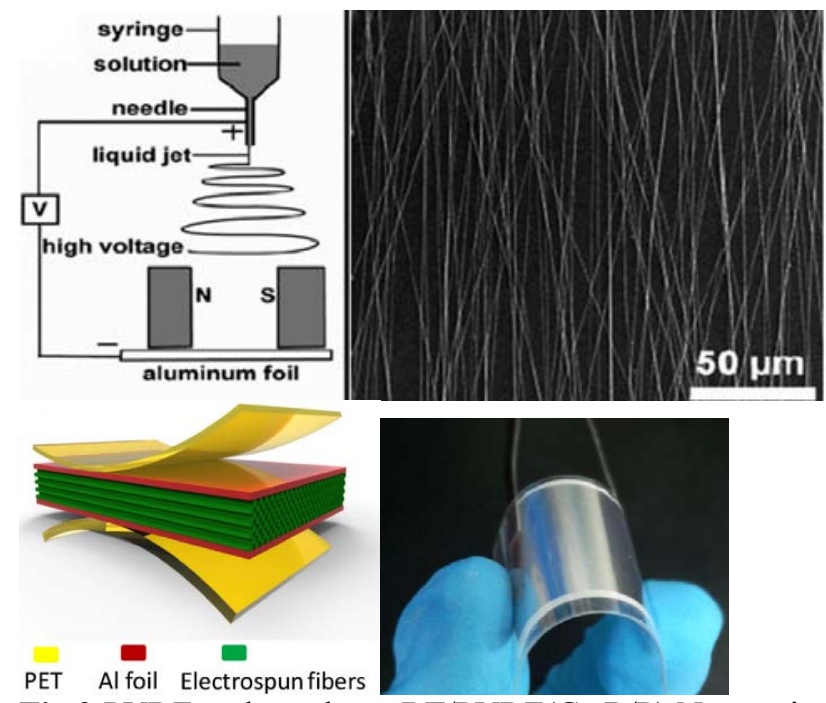

Fig.3 PVDF and graphene-BT/PVDF(Gr-B/P) Nano-wire process and final sensorflexibility diagram[12].

The thin film sensors used in application are designed on the basis of a nanocomposite PVDF/ $\mathrm{BaTiO}_{3} \mathrm{NW}$ fibrous electrospun membrane [9]. The fibers as spun from pure PVDF are smooth with an average diameter of $0.37 \mu \mathrm{m}$, which become rough and non-uniform when the $\mathrm{BaTiO}_{3} \mathrm{NW}$ is incorporated. The $\mathrm{BaTiO}_{3} \mathrm{NW}$ synthesized by hydrothermal reaction has an average length of 4.8 $\mu \mathrm{m}$ and a diameter of $0.27 \mu \mathrm{m}$. Figure $4 \mathrm{a}$ shows the enlarged image of the fibers containing $\mathrm{BaTiO}_{3}$ NWs aligned along the axial direction and electromechanical response both under flexion motion (Figure 4b) and short pulse excitation versus the $\%$ of GNRs incorporated in the whole matrix (Figure 4c).
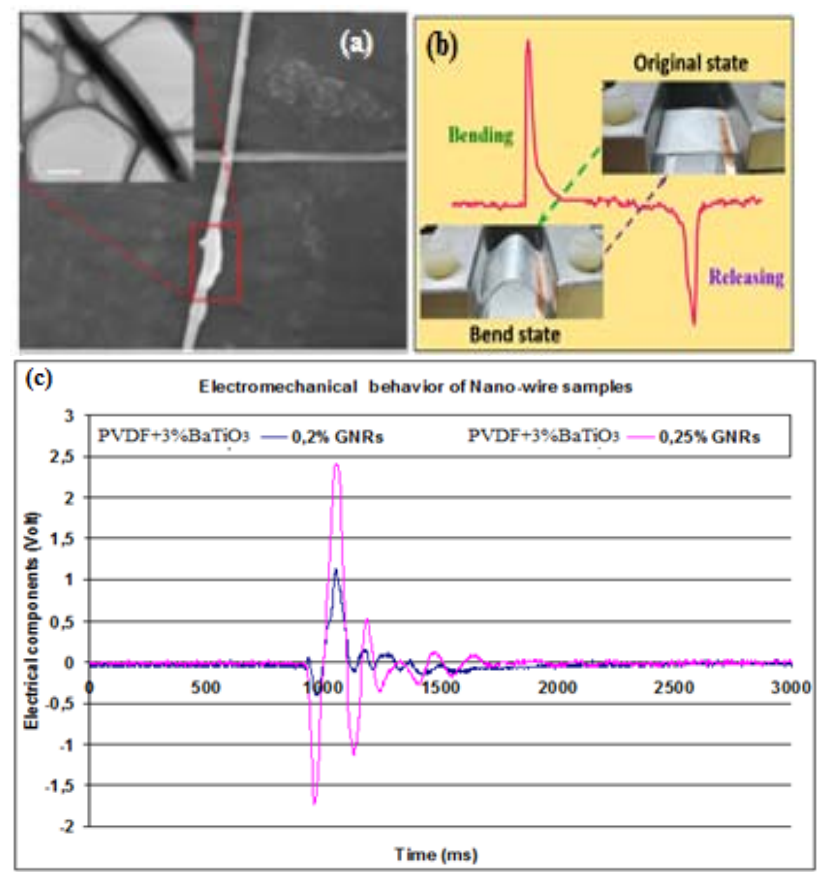

Fig.4 a) High-magnification SEM image of an individual fiber containing $\mathrm{BaTiO}_{3}$ NWs [10]; b) electrical component resulting from a flexural motion of the sensor [10]; c) impulse responses (0.6ms time duration pulse) of the two samples doped with a different $\%$ of GNRs

The works of Sun [8,9] show that the transformation of polymer solutions into electrospun fibers is greatly influenced by many technological parameters. When the proportion of $\mathrm{BaTiO}_{3} \mathrm{NWs}$ is above $3 \mathrm{wt} \%$, the concentration of the precursor exceeds the critical value for electrospinning, and no fibers are formed.

\subsubsection{Experimental results}

In addition to various results appearing in Sun $[8,9]$, Kunming [10] and Wenzhe [11] -works showing the field of potential applications of sensors- we focusedin this study on their integrations in a solid structure resulting from a thermo-deformable material making it possible to satisfy our needs as an intelligent orthosis accessory or in the concept of a reactive exoskeleton. 
Figure 5 shows a principle of application of a reactive intelligent prosthesis by the integration of four sensors in the internal tissues of the solid structure molded on the shape of the handle.

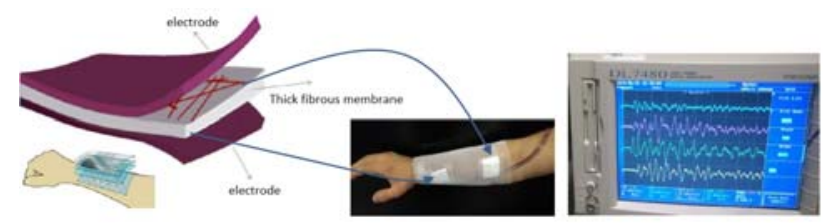

Fig.5 Illustration of the experimental setup for in-vivo sensitivity application.

These receptor sensors are spatially identified with respect to a reference made possible by the trilateration protocol to precisely identify the spatiotemporal location of the mechanical action of which the prosthesis has been the object.

The interest of this application rests both in its ability to follow the movement of muscles in line with the digital approach developed above and to make prosthesis sensitive to sensations coupled to the patient through a graphical unit interface [12] in the case of persons equipped with artificial limbs.

\section{Conclusion}

In this study, we have assessed the need for a more accurate means to quantify the interaction at the interface and in the depth between a substrate (sensor) and biological tissues. A finite element approach associated at an analytical Kelvin-Voigt model have been designed to perform non-invasive measurements, in vivo, of the mechanical behavior of tissues and thus, quantify the dynamic limits of the overall system in order to assess the optimal parameters for an interface coupling properties. The system includes a holding potential to support the dynamics of the members coupled with a real time feeling of the dynamics of the structure.

\section{References:}

[1] Donlan R M and Costerton J W, Biofilms: Survival Mechanisms of Clinically Relevant Microorganisms,ClinicalMicrobiology

Reviews, Vol.15, No.2, 2002, pp.167-193.

[2] Arciola C R, Cenni E, Caramzza R and Pizzoferrato A, Seven surgical silicones retain Staphylococcus aureus differently in vitro,Biomaterials, Vol.16, No.9, 1995, pp.681684.

[3] Millsap K W, Bos R, Van Der Mei H C and BusscherH J, Adhesive interactions between voice prosthetic yeast and bacteria on silicone rubber in the absence and presence of
saliva,Antonie van Leeuwenhoek, Vol.79, No.34, 2001, pp.337-343.

[4] Hutmacher D W, Silver Jubilee Compendium, The Biomaterials, 2000.

[5] Verran $\mathrm{J}$ and Boyd R D, The relationship between substratum surface roughness and microbiological and organic soiling: A review, Biofouling, Vol.17, No.1, 2001, pp.59-71.

[6] Zinger $\mathrm{O}$ et al., Time-dependent morphology and adhesion of osteoblastic cells on titanium model surfaces featuring scale-resolved topography, Biomaterials, Vol.25, No.14, 2004, pp.2695-2711.

[7] Lange R, Lüthen F, Beck U, Rychly J, Baumann A and Nebe B, Fabrication and formation mechanism of closed-loop fibers by electrospinning with a tip collector,Biomolecular engineering, Vol.19, No.2-6, 2002, pp.255-261.

[8] Sun B, Long YZ, Chen ZJ, Liu SH, Zhang HD, Zhang JC and Han WP, Recent advances in flexible and stretchable electronic devices via electrospinning,Journal of Materials Chemistry C, Vol.2, No.7, 2014, pp.1209-1219.

[9] Sun B, Jiang XJ, Zhang S, Zhang JC, Li YF, You QZ and Long YZ, Electrospun anisotropic architectures and porous structures for tissue engineering,Journal of Materials Chemistry B, Vol.3, No.27, 2015, pp.5389-5410.

[10] Sun B, Jiang XJ, Zhang S, Zhang JC, Li YF, You QZ and Long YZ, Electrospun anisotropic architectures and porous structures for tissue engineering,Journal of Materials Chemistry B, Vol.3, No.27, 2015, pp.5389-5410.

[11] Shi K, Sun B, Huang $X$ and Jiang $P$, Synergistic effect of graphene nanosheet and $\mathrm{BaTiO} 3$ nanoparticles on performance enhancement of electrospun PVDF nanofiber mat for flexible piezoelectric nanogenerators,Nano Energy, Vol.52, 2018, pp.153-162.

[12] Guo W, Tan C, Shi K, Li J, Wang XX, Sun B, Huang $\mathrm{X}$, Long $\mathrm{YZ}$ and Jiang $\mathrm{P}$, Wireless piezoelectric devices based on electrospun $\mathrm{PVDF} / \mathrm{BaTiO} 3 \mathrm{NW}$ nanocomposite fibers for human motion monitoring,Nanoscale, Vol.10,No.37, 2018, pp.17751-17760.

[13] [12] Nemmich A, Benabdallah $M$, Hamlil M, Rerbal S and Nassar G, Development of a platform dedicated to the telemedical practice under local architecture USB-HID and distant TCP-IP,International Journal of Engineering \& Technology, Vol.15,No.3, 2015, pp.20-27. 